\title{
Effects of hyperoxemia on aneurysmal subarachnoid hemorrhage outcomes: a systematic review and meta-analysis
}

\author{
Jungeun Ahn, BS, ${ }^{1}$ Panagiotis Mastorakos, MD, PhD, ${ }^{2}$ Jennifer D. Sokolowski, MD, PhD, ${ }^{2}$ \\ Ching-Jen Chen, MD, ${ }^{3}$ Ryan Kellogg, MD, ${ }^{2}$ and Min S. Park, MD ${ }^{2}$
}

${ }^{1}$ School of Medicine, University of Virginia, Charlottesville, Virginia; ${ }^{2}$ Department of Neurosurgery, University of Virginia Health System, Charlottesville, Virginia; and 'Department of Neurosurgery, Thomas Jefferson University Hospital, Philadelphia, Pennsylvania

OBJECTIVE In recent years, hyperoxemia in the intensive care unit has received attention as potentially contributing to negative outcomes in the setting of cardiac arrest, ischemic stroke, and traumatic brain injury. The authors sought to evaluate whether hyperoxemia contributes to worse outcomes in the setting of aneurysmal subarachnoid hemorrhage (aSAH) and to summarize suggested pathophysiological mechanisms.

METHODS A systematic literature review was conducted without date restrictions on the PubMed and Web of Science databases on September 15, 2021. All studies that assessed the relationship between patients treated for aSAH and hyperoxemia were eligible independent of the criteria used to define hyperoxemia. All nonclinical studies and studies that did not report outcome data specific to patients with aSAH were excluded. A total of 102 records were found and screened, resulting in assessment of 10 full-text studies, of which 7 met eligibility criteria. Risk of bias was assessed using the Downs and Black checklist. A meta-analysis on the pooled 2602 patients was performed, and forest plots were constructed. Additionally, a review of the literature was performed to summarize available data regarding the pathophysiology of hyperoxemia.

RESULTS The included studies demonstrated an association between hyperoxemia and increased morbidity and mortality following aSAH. The criteria used to determine hyperoxemia varied among studies. Pooling of univariate data showed hyperoxemia to be associated with poor neurological outcome (OR 2.26, 95\% Cl 1.66-3.07; $p<0.001)$, delayed cerebral ischemia $(\mathrm{DCl})(\mathrm{OR} 1.91,95 \% \mathrm{Cl} 1.31-2.78 ; \mathrm{p}<0.001)$, and increased incidence of poor neurological outcome or mortality as a combined endpoint (OR $2.36,95 \% \mathrm{Cl} 1.87-2.97 ; p<0.001)$. Pooling of multivariable effect sizes showed the same relationship for poor neurological outcome (OR 1.28, 95\% $\mathrm{Cl} 1.07-1.55 ; p=0.01)$ and poor neurological outcome and mortality as a combined endpoint (OR 1.17, 95\% Cl 1.11-1.23; $p<0.001)$. Additionally, review of preclinical studies underlined the contribution of oxidative stress due to hyperoxemia to acute secondary brain injury and $\mathrm{DCl}$.

CONCLUSIONS Reported outcomes from the available studies have indicated that hyperoxemia is associated with worse neurological outcome, mortality, and $\mathrm{DCl}$. These findings provide a general guideline toward avoiding hyperoxemia in the acute setting of aSAH. Further studies are needed to determine the optimal ventilation and oxygenation parameters for acute management of this patient population.

https://thejns.org/doi/abs/10.3171/2021.12.FOCUS21660

KEYWORDS aneurysmal subarachnoid hemorrhage; hyperoxemia; oxidative stress; vasospasm

$\Lambda$ NEURYSMAL subarachnoid hemorrhage (aSAH) is a devastating event that accounts for $3 \%$ to $5 \%$ of all strokes and has a 30-day mortality of 30\%.$^{1,2}$ Following survival of the initial event and securing of the aneurysm, the primary contributor of disability and death is cerebral vasospasm, which results in delayed cerebral ischemia (DCI) and infarction. ${ }^{3}$ Despite the improvement of intensive care unit (ICU) patient management, widespread use of nimodipine, and development of endovascular approaches for vasospasm treatment, morbidity and mortality associated with cerebral vasospasm persist. ${ }^{3}$ The pathophysiology of vasospasm is still only partially understood and precise spasmogenic components of SAH have yet to be established. ${ }^{4,5}$

ABBREVIATIONS aSAH = aneurysmal subarachnoid hemorrhage; $\mathrm{DCl}=$ delayed cerebral ischemia; $\mathrm{ES}=$ effect size; $\mathrm{ICU}=$ intensive care unit; $\mathrm{ROS}=$ reactive oxygen species; TBI = traumatic brain injury; TWA = time-weighted average.

SUBMITTED October 31, 2021. ACCEPTED December 10, 2021.

INCLUDE WHEN CITING DOI: 10.3171/2021.12.FOCUS21660. 
Supplemental oxygen is ubiquitous in the acute setting during the treatment of critically ill patients, including those with aSAH. Oxygen therapy frequently is reflexively used or continued without comprehensive evaluation, ${ }^{6}$ and hyperoxemia tends to be tolerated. ${ }^{7}$ However, hyperoxemia has been associated with poor outcomes in the setting of ischemic stroke, traumatic brain injury (TBI), and post-cardiac arrest..$^{8-11}$ There is growing literature suggesting an association between early hyperoxemia following aSAH with DCI and poor outcomes. ${ }^{11-17}$

Several mechanisms connecting hyperoxemia with poor outcomes following aSAH have been proposed. Supraphysiological levels of oxygen result in production of reactive oxygen species (ROS), leading to oxidative stress and cerebral inflammation, neuronal death, blood-brain barrier breakdown, and reduction in cerebral blood flow. ${ }^{18}$ This contributes to early brain injury after SAH as well as DCI.

Examining the effects of hyperoxemia on aSAH is critical to improve intensive care of patients with SAH. In this review, we assessed the available clinical literature and conducted a meta-analysis to evaluate the association between hyperoxemia and outcomes in the setting of aSAH.

\section{Methods}

A registered protocol was not used for this review. PubMed and Web of Science databases were searched for clinical studies (randomized controlled trials, retrospective or prospective cohort studies, and case series) on September 15, 2021, using the search terms "hyperoxemia OR hyperoxia" AND "subarachnoid hemorrhage OR intracranial hemorrhage." No publication date restrictions were imposed. Duplicates were removed after review of full texts. Titles, abstracts, and sources cited within full texts were independently screened and reviewed for eligibility by two authors.

\section{Study Selection}

Studies that described the relationship between clinical outcomes of patients with aSAH and hyperoxemia exposure were identified based on titles and abstracts. We excluded review articles, letters and replies, preclinical/animal studies, studies conducted on patients without aSAH, studies that did not report clinical outcomes, and studies that did not report data specific to patients with aSAH. We included studies independent of the definition of hyperoxemia exposure.

\section{Data Extraction and Outcome Definitions}

All data were extracted independently by a single author (J.A.) and then reviewed for accuracy by a second author (P.M.). Baseline characteristics extracted included patient age, sex, maximum $\mathrm{PaO}_{2}$, aneurysm location (anterior vs posterior), World Federation of Neurosurgical Societies grade, Fisher grade, and Hunt and Hess grade. All relevant reported outcome data were extracted, including crude data and reported effect sizes (ESs). The reported outcome measures included neurological outcome, incidence of vasospasm and/or DCI, and mortality. ESs were reported as odds ratios ${ }^{11,13-17}$ or risk ratios. ${ }^{12}$ Included studies defined poor neurological outcome at, or 3 months after, discharge based on modified Rankin Scale scores of 3 or 4 to 6 , or Glasgow Outcome Scale scores < 3. If reported, mortality was given as in-hospital mortality or mortality at 3 months. Authors typically defined vasospasm and/or DCI with transcranial Doppler ultrasonography, CTA, or imaging results combined with need for therapy. We constructed mortality or poor neurological outcome as a combined outcome by pooling unadjusted or adjusted ESs of either reported mortality or poor neurological outcome. If a study reported both outcomes, poor neurological outcome was used preferentially. If the authors reported quartiles/quintiles of $\mathrm{PaO}_{2}{ }^{12,14}$ rather than hyperoxia exposure as a binary measure, we opted to use $200 \mathrm{~mm} \mathrm{Hg}$ as the threshold for hyperoxia, as this most closely aligned with existing oxygen bands used by the included studies. If the authors made a distinction between normoxia and hypoxemia cohorts, we used only the normoxia cohort as the reference.

\section{Statistical Analysis}

All statistical analyses were conducted using IBM SPSS Statistics version 28 (IBM Corp.). Available patient demographics of included studies were pooled as a weighted average and pooled standard deviation. Baseline characteristics reported as a median and IQR were converted to mean and standard deviation on the assumption of normal distribution. Aggregate patient demographic information was used if SAH-specific information was not available. ${ }^{11}$ Bias and quality of all included studies were assessed using the Downs and Black checklist. ${ }^{19}$ Metaanalysis was conducted for reported ESs that describe the relationship between hyperoxemia and various reported outcomes. Pooling of crude data or ESs was computed using the restricted maximum likelihood random-effects model for binary variables. ESs were reported as an odds ratio with $95 \%$ confidence interval. Meta-analyses of unadjusted or adjusted ES were constructed into forest plots. Adjusted ESs were pooled as reported without modification. We analyzed study heterogeneity using Cochran's Q and $\mathrm{I}^{2}$ test. Significant heterogeneity was defined as both a $\mathrm{Q}$ value with $\mathrm{p}<0.10$ and the $\mathrm{I}^{2}$ value exceeding $50 \%$. Statistical significance was defined as $p<0.05$.

\section{Results}

Our initial search identified 102 records. After screening of titles and abstracts, 10 full-text articles were reviewed for eligibility (Fig. 1). A total of 7 studies were included for analysis.

Table 1 shows basic characteristics of the 7 included studies published between 2014 and $2021(\mathrm{n}=2602$ patients). All were retrospective, single- or multicenter observational studies. All studies, with one exception, ${ }^{11}$ adjusted reported outcomes of interest for severity of illness. The period of data collection ranged from 1996 to 2018 and the cohort size of each study ranged from 196 to 936 patients. Included studies evaluated for hyperoxia exposure at the time of ICU admission ${ }^{11}$ or in the first 24 hours, ${ }^{12,14,16,17} 72$ hours, ${ }^{13}$ or 6 days ${ }^{15}$ following admission. There was significant heterogeneity in the methodology used to evaluate hyperoxia exposure. Four studies used single $\mathrm{PaO}_{2}$ values to assess for hyperoxemia, ${ }^{11-13,17}$ while 3 studies used time- 


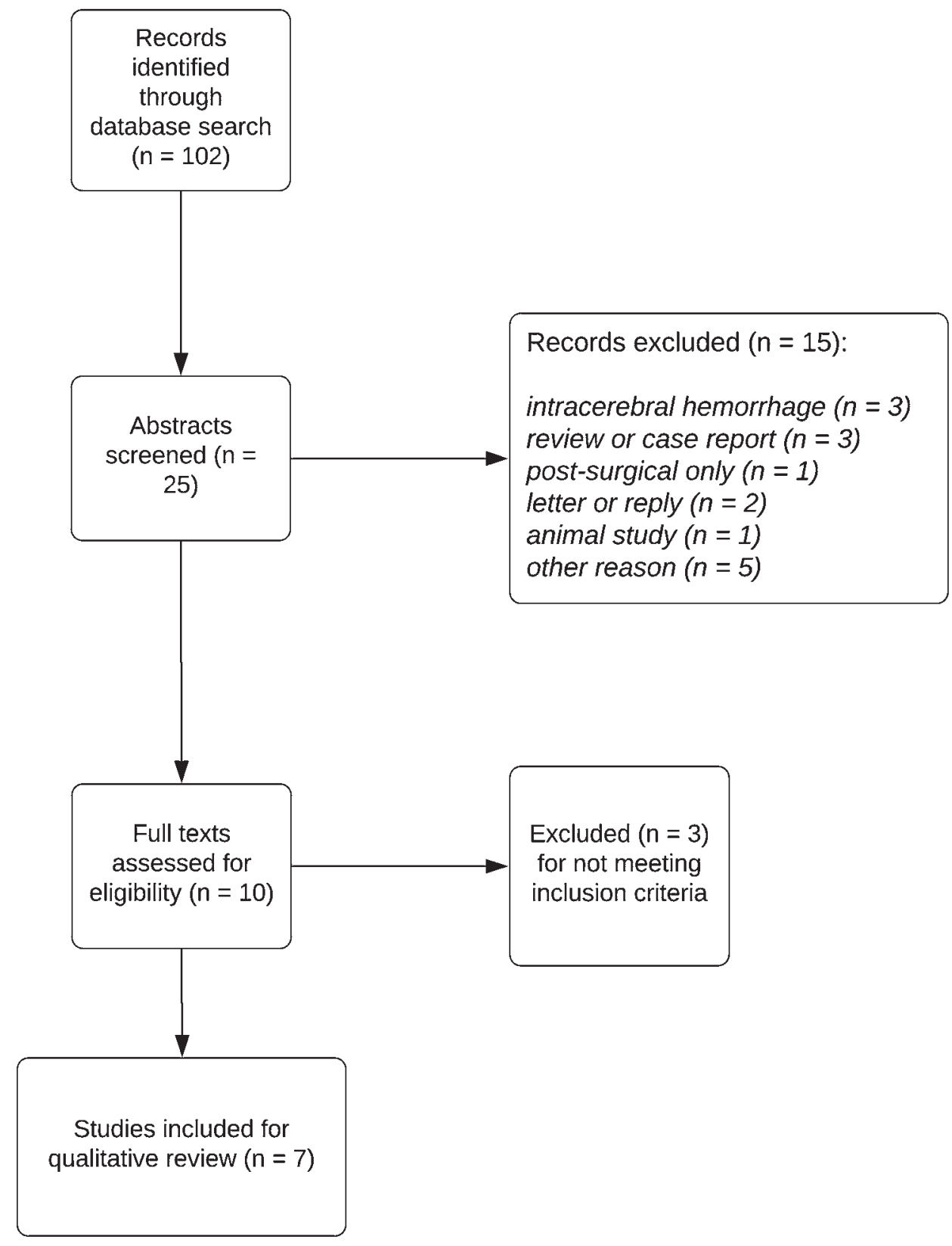

FIG. 1. PRISMA flowchart depicting PubMed and Web of Science literature search.

weighted averages (TWAs) of $\mathrm{PaO}_{2} \cdot{ }^{14-16}$ Table 2 shows the baseline demographic characteristics of the included studies. The mean pooled age of included patients was 58.8 years (SD 14.4 years), and $54.8 \%$ of patients were female.

Table 3 shows the crude outcome data and adjusted ESs extracted from the included studies; there was significant heterogeneity in the types of reported outcomes among them. Five of the 7 selected studies reported neurological outcome at, or 3 months after, hospital discharge. ${ }^{12,14-17}$ Five studies reported DCI or cerebral vasospasm, ${ }^{12-16}$ with 3 of these 5 studies performing multivariate analysis for this outcome measure. ${ }^{13-15}$ Three of the 7 studies reported mortality at 3 months or at discharge, , 13,16 with 2 performing multivariate analysis for this outcome..$^{13,16}$

\section{Pooled Analysis}

The included studies were pooled for further analysis. All were retrospective, single- or multicenter observational studies. Meta-analysis of unadjusted outcome data showed that hyperoxemia was associated with worse neurological outcome (OR 2.26, 95\% CI 1.66-3.07; p < 0.001; Fig. 2A), increased likelihood of mortality or poor neurological outcome measured as a combined endpoint (OR 2.36, 95\% CI 1.87-2.97; p < 0.001; Fig. 2B), and DCI (OR 1.91, 95\% CI 1.31-2.78; $\mathrm{p}<0.001$; Fig. 2C). Meta-analysis of adjusted ESs showed the same relationship for neurological outcome (OR 1.28, 95\% CI 1.07-1.55; $\mathrm{p}=0.01$; Fig. $3 \mathrm{~A})$ and mortality or poor neurological outcome measured as a combined endpoint (OR 1.17, 95\% CI 1.11-1.23; p < 


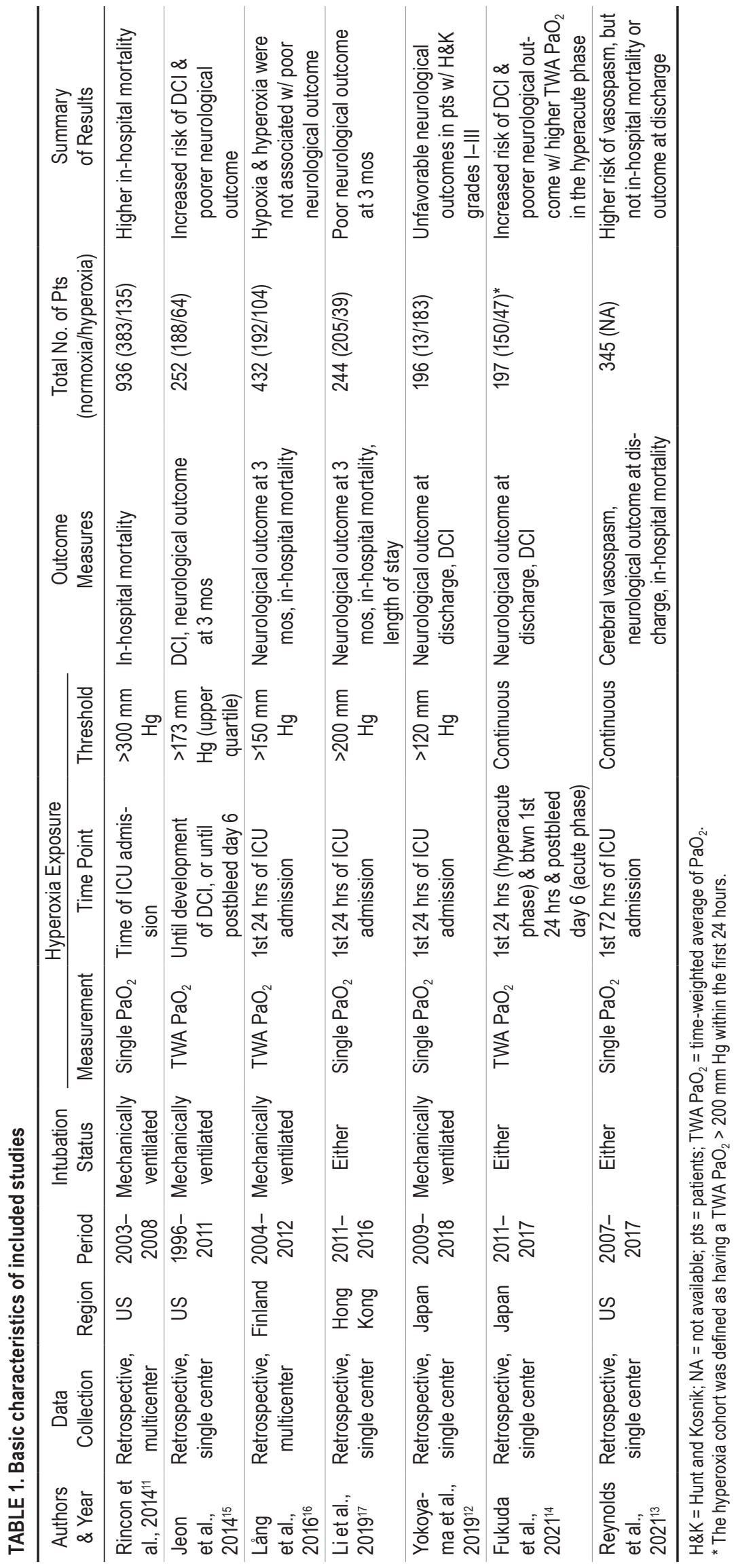


TABLE 2. Demographic and clinical characteristics of included studies

\begin{tabular}{|c|c|c|c|c|c|c|c|c|}
\hline $\begin{array}{l}\text { Authors } \\
\text { \& Year }\end{array}$ & $\begin{array}{l}\text { No. of } \\
\text { Pts }\end{array}$ & $\begin{array}{l}\text { Mean Age, } \\
\text { yrs (SD) }\end{array}$ & $\begin{array}{c}\text { Female Sex, } \\
\text { n (\%) }\end{array}$ & $\begin{array}{c}\text { Mean Maximum } \\
\mathrm{PaO}_{2}, \mathrm{~mm} \\
\mathrm{Hg}(\mathrm{SD})\end{array}$ & $\begin{array}{c}\text { Anterior } \\
\text { Circulation } \\
\text { Aneurysm, } \mathrm{n}(\%)\end{array}$ & $\begin{array}{l}\text { WFNS Grade } \\
\text { IV or V, n (\%) }\end{array}$ & $\begin{array}{c}\text { Fisher Grade } 3 \\
\text { or } 4, \mathrm{n}(\%)\end{array}$ & $\begin{array}{c}\text { Hunt \& } \\
\text { Hess Grade } \\
\text { III-V, n (\%) }\end{array}$ \\
\hline Rincon et al., $2014^{11}$ & $2894^{*}$ & $61(15)$ & $1408(49)$ & $274(148)$ & NA & NA & NA & NA \\
\hline Jeon et al., $2014^{15}$ & 252 & $56.7(13.9)$ & $179(71.0)$ & $146.5(37.0) \dagger$ & NA & NA & $199(79.0)$ & $233(92.5)$ \\
\hline Lång et al., $2016^{16}$ & 432 & $56(11.9)$ & $259(60.0)$ & $126.0(48.4) \dagger$ & $367(85.0)$ & $289(66.8)$ & $392(90.7)$ & $343(79.4)$ \\
\hline Li et al., $2019^{17}$ & 244 & $57.7(14.6)$ & $155(63.5)$ & $151.2(62.6)$ & $153(62.7)$ & $107(43.9)$ & $183(75.0)$ & NA \\
\hline Yokoyama et al., $2019^{12}$ & 196 & $62.7(16.8)$ & $133(67.9)$ & $193(70.4) \dagger$ & NA & NA & NA & NA \\
\hline Fukuda et al., $2021^{14}$ & 197 & $62.1(15.7)$ & $133(67.5)$ & NA & $105(53.3)$ & NA & NA & NA \\
\hline Reynolds et al., $2021^{13}$ & 345 & $54.5(13.4)$ & $234(67.8)$ & $218.8(117.3)$ & NA & NA & $310(89.9)$ & $240(69.6)$ \\
\hline Pooled estimate & 4560 & $58.8(14.4) \ddagger$ & $2501(54.8)$ & $207.1(104.8)$ & $625 / 873(71.6)$ & $396 / 676(58.6)$ & $1084 / 1273(85.2)$ & $816 / 1029$ (79.3) \\
\hline
\end{tabular}

WFNS $=$ World Federation of Neurosurgical Societies

* Study only provided aggregate demographic information for all included patients, including those diagnosed with TBI, intracranial hemorrhage, and SAH.

† Converted to mean (SD) from median and IQR while assuming a normal distribution.

$\ddagger$ Pooled estimates for continuous variables were given as weighted averages and SDs. Data from studies that provided only aggregate data were weighted for the number of patients within the corresponding SAH cohort.

0.001; Fig. 3B), but showed no significant association with DCI (OR 1.47, 95\% CI 0.81-2.67; $\mathrm{p}=0.20$; Fig. 3C). The pooling of multivariate odds ratios for DCI was marked by significant heterogeneity.

\section{Discussion}

Administration of supplemental oxygen constitutes the default approach in the acute setting for critically ill patients. Here, we investigated the effects of hyperoxia on outcomes after aSAH. We systematically identified 7 retrospective clinical studies and performed a meta-analysis, which showed an association between hyperoxia and the risk of poor neurological outcome, mortality, and DCI. Our results underline the need for judicious oxygen supplementation in the acute setting after aSAH.

While hypoxia is often the immediate concern where brain injury is concerned, oxygen itself cannot be considered a benign agent. The potential pulmonary toxicity of oxygen therapy has been well described. ${ }^{20}$ The brain is one of the first organs to experience the effects of hyperoxemia due to the generation of ROS..$^{21,22}$ Supraphysiological levels of oxygen result in production of ROS, leading to oxidative stress, cerebral inflammation, neuronal death, blood-brain barrier breakdown, and reduction in cerebral blood flow. ${ }^{18}$ Oxidative stress has been associated with poor outcomes in the setting of ischemic stroke, TBI, and post-cardiac arrest. ${ }^{8-11}$

Several mechanisms connecting hyperoxemia with poor outcomes following aSAH have been proposed. These include acute effects of oxidative stress on early brain injury and effects of hyperoxemia on DCI..$^{13,23}$ Following acute brain injury, disruption of cellular respiration results in increased production of ROS that overwhelm the antioxidant systems and contribute to secondary brain injury. ${ }^{23,24}$ Indeed, it has been found that even healthy patients exposed to hyperoxia transiently develop increased ROS burden, lipid peroxidation, and reduced nitric oxide metabolites. ${ }^{25}$ Moreover, hyperoxemia promotes oxidation of extravascular cell-free hemoglobin, which is known to be proinflammatory in the setting of SAH and has been associated with vasospasm of the pulmonary arteries. ${ }^{23,26,27}$ It is also believed that ROS may be involved with NLRP3 inflammasome activation ${ }^{28,29}$ and upregulation of oxidized low-density lipoprotein (LDL) and its receptor, lectin-like oxidized LDL receptor- $1 .{ }^{30-32}$ Both are currently being studied for their potential roles in the development of DCI.

TABLE 3. Extracted unadjusted and adjusted outcome data from included studies

\begin{tabular}{|c|c|c|c|c|c|c|c|c|c|}
\hline \multirow[b]{2}{*}{$\begin{array}{l}\text { Authors } \\
\& \text { Year }\end{array}$} & \multicolumn{3}{|c|}{ Poor Neurological Outcome } & \multicolumn{3}{|c|}{$\mathrm{DCl}$ or Cerebral Vasospasm } & \multicolumn{3}{|c|}{ Mortality } \\
\hline & Hyperoxia & Normoxia & $\begin{array}{l}\text { Adjusted ES } \\
(95 \% \mathrm{Cl})\end{array}$ & Hyperoxia & Normoxia & $\begin{array}{l}\text { Adjusted ES } \\
(95 \% \mathrm{Cl})\end{array}$ & Hyperoxia & Normoxia & $\begin{array}{l}\text { Adjusted ES } \\
(95 \% \mathrm{Cl})\end{array}$ \\
\hline Rincon et al., 201411 & NA & NA & NA & NA & NA & NA & $80 / 135$ & $139 / 383$ & NA \\
\hline Jeon et al., $2014^{15}$ & $32 / 18$ & $76 / 76$ & $2.30(1.03-5.12)$ & $36 / 28$ & $61 / 127$ & $3.16(1.69-5.92)$ & NA & NA & NA \\
\hline Lång et al., $2016^{16}$ & $83 / 21$ & $101 / 91$ & $1.09(0.61-1.97)$ & $28 / 108$ & $32 / 160$ & NA & $37 / 67$ & $57 / 135$ & $0.73(0.38-1.40)$ \\
\hline Li et al., $2019^{17}$ & $27 / 12$ & $95 / 110$ & $3.788(1.13-12.70)$ & NA & NA & NA & NA & NA & NA \\
\hline Yokoyama et al., $2019^{12}$ & $48 / 40$ & $42 / 60$ & $1.38(0.99-1.83)$ & $17 / 71$ & $10 / 98$ & NA & NA & NA & NA \\
\hline Fukuda et al., $2021^{14}$ & $25 / 22$ & $57 / 93$ & $1.17(1.06-1.29)$ & $16 / 31$ & $26 / 89$ & $1.09(1.01-1.17)$ & NA & NA & NA \\
\hline Reynolds et al., $2021^{13}$ & NA & NA & NA & NA & NA & $1.15(1.03-1.28)$ & NA & NA & $1.10(0.97-1.25)$ \\
\hline
\end{tabular}

Values represent the number of patients with outcome/patients without outcome data within the hyperoxia or normoxia cohorts, unless indicated otherwise. We used $200 \mathrm{~mm} \mathrm{Hg}$ as the threshold for hyperoxia based on the oxygen bands defined within the study and similar thresholds used in other studies. 


\section{A. Neurologic outcome}

\begin{tabular}{|c|c|c|c|c|c|c|}
\hline Study & OR & Lower & Upper & $\mathrm{p}$-value & Weight & Weight (\%) \\
\hline Jeon et al ${ }^{15}$ & 1.78 & 0.92 & 3.44 & 0.09 & 7.44 & 18.48 \\
\hline Lang et al ${ }^{16}$ & 3.60 & 2.06 & 6.28 & 0.00 & 9.83 & 24.42 \\
\hline Li et $\mathrm{al}^{17}$ & 2.61 & 1.25 & 5.42 & 0.01 & 6.20 & 15.40 \\
\hline Yokoyama et & $\mathrm{tal}^{12} 1.89$ & 1.07 & 3.34 & 0.03 & 9.42 & 23.42 \\
\hline Fukuda et al $^{14}$ & ${ }^{14} 1.71$ & 0.88 & 3.33 & 0.11 & 7.36 & 18.28 \\
\hline Overall & 2.26 & 1.66 & 3.07 & $<0.001$ & & \\
\hline
\end{tabular}

Forest Plot

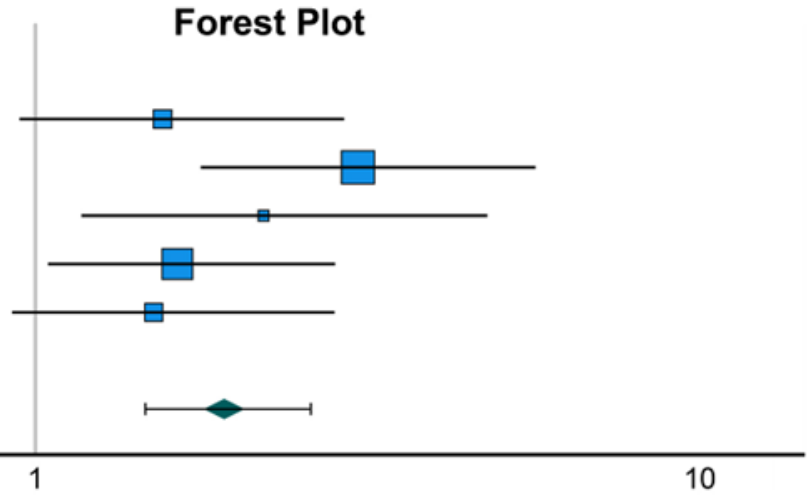

Model: Random-effects model

0

Heterogeneity: Tau-squared $=0.02, \mathrm{H}$-squared $=1.21$, I-squared $=0.17$

Homogeneity: $Q=4.40, d f=4, p$-value $=0.35$

Test of overall effect size: $z=5.16$, p-value $<0.001$

\section{B. Composite outcome}

Study OR Lower Upper p-value Weight Weight (\%)

$\begin{array}{llllll}\text { Rincon et } \text { al }^{11} 2.55 & 1.71 & 3.81 & 0.00 & 23.82 & 32.74\end{array}$

$\begin{array}{lllllll}\text { Jeon et al }^{15} & 1.78 & 0.92 & 3.44 & 0.09 & 8.84 & 12.15\end{array}$

$\begin{array}{lllllll}\text { Lang et al }^{16} & 3.60 & 2.06 & 6.28 & 0.00 & 12.43 & 17.09\end{array}$

$\begin{array}{lllllll}\text { Li et al }^{17} & 2.61 & 1.25 & 5.42 & 0.01 & 7.14 & 9.82\end{array}$

$\begin{array}{llllll}\text { Yokoyama et al } 1.89 & 1.07 & 3.34 & 0.03 & 11.79 & 16.21\end{array}$

$\begin{array}{llllll}\text { Fukuda et } \mathrm{al}^{14} 1.71 & 0.88 & 3.33 & 0.11 & 8.72 & 11.99\end{array}$

$\begin{array}{lllll}\text { Overall } & 2.36 & 1.87 & 2.97 & <0.001\end{array}$

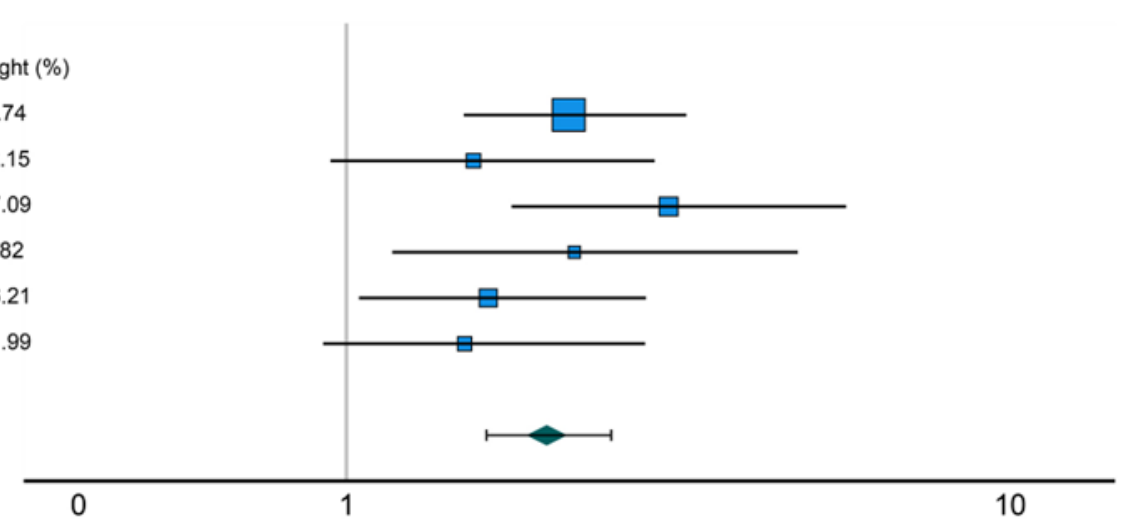

0

0

Model: Random-effects model

Heterogeneity: Tau-squared $=0.00, \mathrm{H}$-squared $=1.00$, l-squared $=0.00$

Homogeneity: $Q=4.63, \mathrm{df}=5, \mathrm{p}$-value $=0.46$

Test of overall effect size: $z=7.31, p$-value $<0.001$

\section{DCI}

\begin{tabular}{|c|c|c|c|c|c|c|}
\hline Study & OR & Lower & Upper & p-value & Weight & Weight (\%) \\
\hline Jeon et al ${ }^{15}$ & 2.68 & 1.50 & 4.78 & 0.00 & 8.21 & 30.41 \\
\hline Lang et $\mathrm{al}^{16}$ & 1.30 & 0.74 & 2.28 & 0.37 & 8.58 & 31.79 \\
\hline Yokoyama et & al 2.35 & 1.01 & 5.43 & 0.05 & 4.61 & 17.05 \\
\hline Fukuda et al $^{1}$ & 41.77 & 0.84 & 3.72 & 0.13 & 5.60 & 20.75 \\
\hline Overall & 1.91 & 1.31 & 2.78 & $<0.001$ & & \\
\hline
\end{tabular}

Model: Random-effects model

0

Heterogeneity: Tau-squared $=0.03, \mathrm{H}$-squared $=1.30$, l-squared $=0.23$

Homogeneity: $\mathrm{Q}=3.39, \mathrm{df}=3, \mathrm{p}$-value $=0.34$

Test of overall effect size: $z=3.35, p$-value $<0.001$

.




\section{A. Neurologic outcome}

$\begin{array}{lcccccc}\text { Study } & \text { OR } & \text { Lower } & \text { Upper } & \text { p-value } & \text { Weight } & \text { Weight (\%) } \\ \text { Jeon et al } & 2.30 & 1.03 & 5.13 & 0.04 & 5.52 & 4.97 \\ \text { Lang et al }^{16} & 1.09 & 0.61 & 1.96 & 0.77 & 9.68 & 8.72 \\ \text { Li et al }^{17} & 3.79 & 1.13 & 12.69 & 0.03 & 2.53 & 2.28 \\ \text { Yokoyama et al } 1.38 & 1.02 & 1.88 & 0.04 & 26.02 & 23.44 \\ \text { Fukuda et al } & 1.17 & 1.10 & 1.24 & 0.00 & 67.28 & 60.59 \\ & & & & & & \\ \text { Overall } & 1.28 & 1.07 & 1.55 & 0.01 & & \end{array}$

\section{Forest Plot}

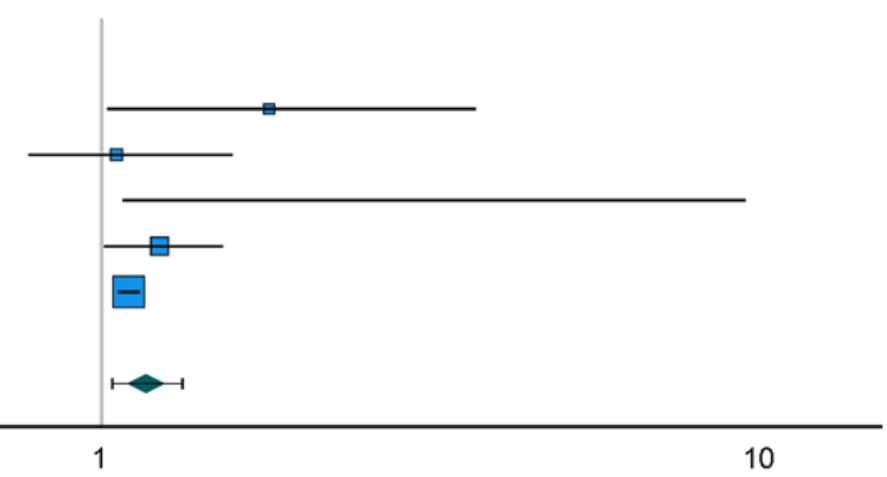

Model: Random-effects model

Heterogeneity: Tau-squared $=0.01, \mathrm{H}$-squared $=1.40, \mathrm{l}$-squared $=0.29$

Homogeneity: $\mathrm{Q}=7.36, \mathrm{df}=4, \mathrm{p}$-value $=0.12$

Test of overall effect size: $z=2.63$, $p$-value 0.01

\section{B. Composite outcome}

\begin{tabular}{|c|c|c|c|c|c|c|}
\hline Study & OR & Lower & Upper & p-value & Weight & Weight (\%) \\
\hline Jeon et al ${ }^{15}$ & 2.30 & 1.03 & 5.13 & 0.04 & 5.97 & 0.47 \\
\hline Lang et al ${ }^{16}$ & 1.09 & 0.61 & 1.96 & 0.77 & 11.18 & 0.87 \\
\hline Li et al ${ }^{17}$ & 3.79 & 1.13 & 12.69 & 0.03 & 2.63 & 0.21 \\
\hline Yokoyama et & $\mathrm{al}^{12} 1.38$ & 1.02 & 1.88 & 0.04 & 40.68 & 3.18 \\
\hline Fukuda et al ${ }^{1}$ & 41.71 & 1.10 & 1.24 & 0.00 & 980.43 & 76.67 \\
\hline Reynolds et a & $a^{13} 1.10$ & 0.97 & 1.25 & 0.14 & 237.828 & 18.60 \\
\hline
\end{tabular}

$\begin{array}{lllll}\text { Overall } & 1.17 & 1.11 & 1.23 & <0.001\end{array}$

\section{0}

Model: Random-effects model

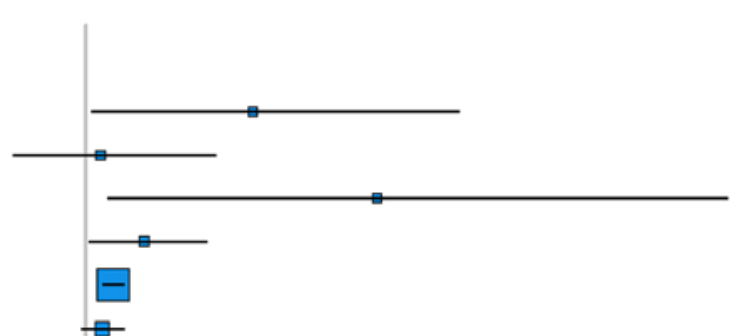

Heterogeneity: Tau-squared $=0.00, \mathrm{H}$-squared $=1.00, \mathrm{l}$-squared $=0.00$

Homogeneity: $\mathrm{Q}=8.43, \mathrm{df}=5, \mathrm{p}$-value $=0.13$

Test of overall effect size: $z=5.57$, p-value $<0.001$

\section{C. $\mathrm{DCl}$}

$\begin{array}{lcccccc}\text { Study } & \text { OR } & \text { Lower } & \text { Upper } & \text { p-value } & \text { Weight } & \text { Weight }(\%) \\ \text { Jeon et al }{ }^{15} & 3.16 & 1.69 & 5.91 & 0.00 & 2.85 & 26.34 \\ \text { Fukuda et al }^{14} 1.09 & 1.01 & 1.17 & 0.02 & 3.99 & 36.95 \\ \text { Reynolds et al } 1.15 & 1.03 & 1.28 & 0.01 & 3.97 & 36.71\end{array}$

Overall $\quad 1.47 \quad 0.81 \quad 2.67 \quad 0.20$

Model: Random-effects model

Heterogeneity: Tau-squared $=0.25, \mathrm{H}$-squared $=58.48, \mathrm{l}$-squared $=0.98$

Homogeneity: $\mathrm{Q}=11.27, \mathrm{df}=2, \mathrm{p}$-value $=0.00$

Test of overall effect size: $z=1.27, p$-value $=0.20$

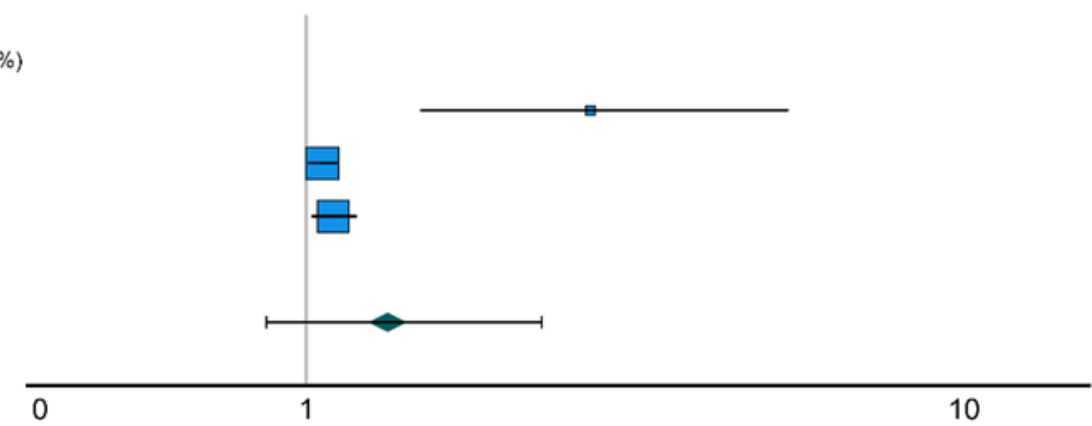

1010

FIG. 3. Forest plots of individual and pooled adjusted ORs for poor neurological outcome (A), mortality or poor neurological outcome as a combined outcome measure (B), and incidence of $\mathrm{DCl}(\mathrm{C})$. Composite outcome was constructed by pooling the reported adjusted ORs for either mortality or poor neurological outcome. If a study reported both outcomes, poor neurological outcome was used preferentially. ORs $>1$ (right side of plot) indicate a positive association between hyperoxemia and the outcome in question. Pooled ORs were calculated with a random-effects model.

ies have shown hyperventilation or hypocapnia to be independently associated with worse outcomes after SAH. ${ }^{17,38,39}$ Further studies are needed to investigate hypocapnia in the context of SAH as well as other pathological processes. ${ }^{40}$
Overall, the mechanisms described suggest that normoxia and normocapnia should be targeted when managing patients with SAH and that hyperoxemia may lead to worse secondary injury and DCI. As expected, due to the 
underlying pathophysiological mechanisms described, the retrospective studies available in the literature have suggested an association between hyperoxia and the risk of poor neurological outcome, mortality, and DCI.

There remains considerable debate in the literature about what constitutes hyperoxemia. ${ }^{8,15,41}$ Correspondingly, there was significant heterogeneity in the methodology used to measure hyperoxemia exposure. Four studies used single $\mathrm{PaO}_{2}$ measurements to determine hyperoxia exposure, while 3 used TWAs. TWA $\mathrm{PaO}_{2}$ measurements may provide an advantage in that many instances of hyperoxia in the ICU may be transient, and, unlike single $\mathrm{PaO}_{2}$ measurements, TWA $\mathrm{PaO}_{2}$ is not affected by the frequency of measurements. Additionally, TWA $\mathrm{PaO}_{2}$ has been statistically correlated to single $\mathrm{PaO}_{2}$ measurements in a previous study that investigated hyperoxia in the setting of TBI. ${ }^{42}$

The predetermined thresholds for hyperoxia exposure among included studies ranged from $120 \mathrm{~mm} \mathrm{Hg} \mathrm{PaO}$ to $300 \mathrm{~mm} \mathrm{Hg} \mathrm{PaO}$. In the study by Fukuda et al., ${ }^{14}$ the median values of 24-hour TWA $\mathrm{PaO}_{2}$ in the DCI and unfavorable outcome groups were 186 (range 141-213) mm Hg and 176 (range 154-205) $\mathrm{mm} \mathrm{Hg}$, respectively. This very closely approximates the $173-\mathrm{mm} \mathrm{Hg} \mathrm{PaO}$ threshold used to define hyperoxia exposure by Jeon et al..$^{15}$ Reynolds et al. ${ }^{13}$ also reported that the mean maximum $\mathrm{PaO}_{2} \pm \mathrm{SD}$ in patients who experienced vasospasm was $232 \mathrm{~mm} \mathrm{Hg} \pm$ $124.1 \mathrm{~mm} \mathrm{Hg}$. In contrast, the threshold $\mathrm{PaO}_{2}$ values used by Yokoyama et al. ${ }^{12}$ and Lång et al..$^{16}$ were much lower at $120 \mathrm{~mm} \mathrm{Hg}$ and $150 \mathrm{~mm} \mathrm{Hg}$, respectively. This may partially explain the variability in results. Importantly, these results suggest that the threshold for hyperoxia that contributes to worse outcomes may lie within the region of $175 \mathrm{~mm} \mathrm{Hg}$ to $200 \mathrm{~mm} \mathrm{Hg}$. It remains unclear whether this effect is dominated by acute or persistent exposure to hyperoxia, although most findings have focused on the initial 24-hour period. To our knowledge, there currently remains no laboratory data to help guide what should be defined as hyperoxia in the setting of SAH. Even animal models have not shown agreement for what degree of hyperoxia, if any, may be deleterious with respect to reperfusion injury. ${ }^{43}$

DCI was found to be associated with hyperoxia after pooling of univariate ESs. However, the meta-analysis of adjusted ES for DCI was characterized by significant heterogeneity. This may be partially explained by differences in patient cohorts. The patients within the cohort of the study by Jeon et al. were mechanically intubated, and thus more likely to be severely ill (Table 1$).{ }^{15}$ In contrast, the studies by Fukuda et al. and Reynolds et al. did not exclude nonintubated patients..$^{14,13}$ Correspondingly, the latter studies reported modest ESs relative to the former.

These findings indicate that hyperoxia in the early periods of ICU admission for SAH is associated with worse outcomes, possibly more pronounced with lower-grade hemorrhages. However, the heterogeneity of the study findings weakens this assertion. Furthermore, it remains less clear how this would translate to clinical practice and what thresholds of $\mathrm{PaO}_{2}$ or TWA $\mathrm{PaO}_{2}$ should be used to guide therapy. There remains a need for prospective studies with greater control of $\mathrm{PaO}_{2}$ levels to further investigate the effects of hyperoxemia on SAH outcomes.

\section{Limitations}

The limitations of this work include the retrospective nature of all included studies and the significant heterogeneity of the study findings with respect to hyperoxia exposure criteria and reported outcomes with mixed availability of crude and adjusted data. Additionally, a majority of the studies were performed at a single center with large ranges of maximum $\mathrm{PaO}_{2}$ within each cohort, reducing the external validity of the studies' findings. While most included studies performed multivariate analysis to account for confounders, residual confounders are an inherent risk of retrospective analysis.

\section{Conclusions}

In accordance with the findings on ischemic stroke, TBI, and post-cardiac arrest, the literature review and meta-analysis conducted here suggests that hyperoxemia may worsen the outcome of patients with aSAH. This is attributed to acute effects of oxidative stress on early brain injury and effects of hyperoxia on DCI. While these findings provide a general guideline toward avoiding hyperoxia in the acute setting of aSAH, further studies are needed to determine the optimal ventilation and oxygenation parameters for acute management of this patient population.

\section{References}

1. Vergouwen MDI, Jong-Tjien-Fa AV, Algra A, Rinkel GJE. Time trends in causes of death after aneurysmal subarachnoid hemorrhage: a hospital-based study. Neurology. 2016; 86(1):59-63.

2. Etminan N, Chang HS, Hackenberg K, et al. Worldwide incidence of aneurysmal subarachnoid hemorrhage according to region, time period, blood pressure, and smoking prevalence in the population: a systematic review and meta-analysis. JAMA Neurol. 2019;76(5):588-597.

3. Al-Khindi T, Macdonald RL, Schweizer TA. Cognitive and functional outcome after aneurysmal subarachnoid hemorrhage. Stroke. 2010;41(8):e519-e536.

4. Zimmermann M, Seifert V. Endothelin and subarachnoid hemorrhage: an overview. Neurosurgery. 1998;43(4):863-876.

5. Pasqualin A. Epidemiology and pathophysiology of cerebral vasospasm following subarachnoid hemorrhage. J Neurosurg Sci. 1998;42(1)(suppl 1):15-21.

6. Parke RL, Eastwood GM, McGuinness SP. Oxygen therapy in non-intubated adult intensive care patients: a point prevalence study. Crit Care Resusc. 2013;15(4):287-293.

7. de Graaff AE, Dongelmans DA, Binnekade JM, de Jonge E. Clinicians' response to hyperoxia in ventilated patients in a Dutch ICU depends on the level of $\mathrm{FiO}_{2}$. Intensive Care Med. 2011;37(1):46-51.

8. Kilgannon JH, Jones AE, Shapiro NI, et al. Association between arterial hyperoxia following resuscitation from cardiac arrest and in-hospital mortality. JAMA. 2010;303(21):2165-2171.

9. Helmerhorst HJF, Roos-Blom MJ, van Westerloo DJ, de Jonge E. Association between arterial hyperoxia and outcome in subsets of critical illness: a systematic review, metaanalysis, and meta-regression of cohort studies. Crit Care Med. 2015;43(7):1508-1519.

10. Brenner M, Stein D, Hu P, Kufera J, Wooford M, Scalea T. Association between early hyperoxia and worse outcomes after traumatic brain injury. Arch Surg. 2012;147(11):1042-1046.

11. Rincon F, Kang J, Maltenfort M, et al. Association between hyperoxia and mortality after stroke: a multicenter cohort study. Crit Care Med. 2014;42(2):387-396. 
12. Yokoyama S, Hifumi T, Kawakita K, Tamiya T, Minamino $\mathrm{T}$, Kuroda Y. Early hyperoxia in the intensive care unit is significantly associated with unfavorable neurological outcomes in patients with mild-to-moderate aneurysmal subarachnoid hemorrhage. Shock. 2019;51(5):593-598.

13. Reynolds RA, Amin SN, Jonathan SV, et al. Hyperoxemia and cerebral vasospasm in aneurysmal subarachnoid hemorrhage. Neurocrit Care. 2021;35(1):30-38.

14. Fukuda S, Koga Y, Fujita M, et al. Hyperoxemia during the hyperacute phase of aneurysmal subarachnoid hemorrhage is associated with delayed cerebral ischemia and poor outcome: a retrospective observational study. J Neurosurg. 2021;134(1): 25-32.

15. Jeon SB, Choi HA, Badjatia N, et al. Hyperoxia may be related to delayed cerebral ischemia and poor outcome after subarachnoid haemorrhage. J Neurol Neurosurg Psychiatry. 2014;85(12):1301-1307.

16. Lång M, Raj R, Skrifvars MB, et al. Early moderate hyperoxemia does not predict outcome after aneurysmal subarachnoid hemorrhage. Neurosurgery. 2016;78(4):540-545.

17. Li KC, Tam CWY, Shum HP, Yan WW. Impact of hyperoxia and hypocapnia on neurological outcomes in patients with aneurysmal subarachnoid hemorrhage: a retrospective study. Crit Care Res Pract. 2019;2019:7584573.

18. Becker LB. New concepts in reactive oxygen species and cardiovascular reperfusion physiology. Cardiovasc Res. 2004; 61(3):461-470.

19. Downs SH, Black N. The feasibility of creating a checklist for the assessment of the methodological quality both of randomised and non-randomised studies of health care interventions. J Epidemiol Community Health. 1998;52(6):377-384.

20. Kallet RH, Branson RD. Should oxygen therapy be tightly regulated to minimize hyperoxia in critically ill patients? Respir Care. 2016;61(6):801-817.

21. Gerschman R, Gilbert DL, Nye SW, Dwyer P, Fenn WO. Oxygen poisoning and $\mathrm{x}$-irradiation: a mechanism in common. Science. 1954;119(3097):623-626.

22. Turrens JF. Mitochondrial formation of reactive oxygen species. J Physiol. 2003;552(Pt 2):335-344.

23. Jelinek M, Jurajda M, Duris K. The role of oxidative stress in early brain injury after subarachnoid hemorrhage. Oxid Med Cell Longev. 2020;2020:8877116.

24. Watts ME, Pocock R, Claudianos C. Brain energy and oxygen metabolism: emerging role in normal function and disease. Front Mol Neurosci. 2018;11:216.

25. Bosco G, Paganini M, Giacon TA, et al. Oxidative stress and inflammation, microRNA, and hemoglobin variations after administration of oxygen at different pressures and concentrations: a randomized trial. Int J Environ Res Public Health. 2021;18(18):9755.

26. Shaver CM, Wickersham N, McNeil JB, et al. Cell-free hemoglobin promotes primary graft dysfunction through oxidative lung endothelial injury. JCI Insight. 2018;3(2):98546.

27. Khey KMW, Huard A, Mahmoud SH. Inflammatory pathways following subarachnoid hemorrhage. Cell Mol Neurobiol. 2020;40(5):675-693.

28. Minutoli L, Puzzolo D, Rinaldi M, et al. ROS-mediated NLRP3 inflammasome activation in brain, heart, kidney, and testis ischemia/reperfusion injury. Oxid Med Cell Longev. 2016;2016:2183026.

29. Dodd WS, Noda I, Martinez M, Hosaka K, Hoh BL. NLRP3 inhibition attenuates early brain injury and delayed cerebral vasospasm after subarachnoid hemorrhage. J Neuroinflammation. 2021;18(1):163.

30. Matsuda N, Ohkuma H, Naraoka M, Munakata A, Shimamura N, Asano K. Role of oxidized LDL and lectin-like oxidized LDL receptor-1 in cerebral vasospasm after subarachnoid hemorrhage. J Neurosurg. 2014;121(3):621-630.
31. Lin Q, Ba HJ, Dai JX, et al. Serum soluble lectin-like oxidized low-density lipoprotein receptor-1 as a biomarker of delayed cerebral ischemia after aneurysmal subarachnoid hemorrhage. Brain Behav. 2020;10(2):e01517.

32. Inoue $\mathrm{T}$, Ishida $\mathrm{T}$, Inoue $\mathrm{T}$, et al. Lectin-like oxidized lowdensity lipoprotein receptor-1 levels as a biomarker of acute intracerebral hemorrhage. J Stroke Cerebrovasc Dis. 2019; 28(2):490-494.

33. Stamler JS, Jia L, Eu JP, et al. Blood flow regulation by S-nitrosohemoglobin in the physiological oxygen gradient. Science. 1997;276(5321):2034-2037.

34. Floyd TF, Clark JM, Gelfand R, et al. Independent cerebral vasoconstrictive effects of hyperoxia and accompanying arterial hypocapnia at 1 ATA. J Appl Physiol (1985). 2003;95(6): 2453-2461.

35. Harper AM, Glass HI. Effect of alterations in the arterial carbon dioxide tension on the blood flow through the cerebral cortex at normal and low arterial blood pressures. J Neurol Neurosurg Psychiatry. 1965;28(5):449-452.

36. Watson NA, Beards SC, Altaf N, Kassner A, Jackson A. The effect of hyperoxia on cerebral blood flow: a study in healthy volunteers using magnetic resonance phase-contrast angiography. Eur J Anaesthesiol. 2000;17(3):152-159.

37. Mattos JD, Campos MO, Rocha MP, et al. Human brain blood flow and metabolism during isocapnic hyperoxia: the role of reactive oxygen species. J Physiol. 2019;597(3):741-755.

38. Williamson CA, Sheehan KM, Tipirneni R, et al. The association between spontaneous hyperventilation, delayed cerebral ischemia, and poor neurological outcome in patients with subarachnoid hemorrhage. Neurocrit Care. 2015;23(3):330-338.

39. Yokoyama S, Hifumi T, Okazaki T, et al. Association of abnormal carbon dioxide levels with poor neurological outcomes in aneurysmal subarachnoid hemorrhage: a retrospective observational study. J Intensive Care. 2018;6(1):83.

40. Roberts BW, Karagiannis P, Coletta M, Kilgannon JH, Chansky ME, Trzeciak S. Effects of PaCO2 derangements on clinical outcomes after cerebral injury: a systematic review. Resuscitation. 2015;91:32-41.

41. Damiani E, Adrario E, Girardis M, et al. Arterial hyperoxia and mortality in critically ill patients: a systematic review and meta-analysis. Crit Care. 2014;18(6):711.

42. Raj R, Bendel S, Reinikainen M, et al. Hyperoxemia and long-term outcome after traumatic brain injury. Crit Care. 2013;17(4):R177.

43. Lehmann RK, Brounts LR, Lesperance KE, et al. Hypoxemic versus normoxemic reperfusion in a large animal model of severe ischemia-reperfusion injury. J Surg Res. 2011;166(2): 194-198.

\section{Disclosures}

The authors report no conflict of interest concerning the materials or methods used in this study or the findings specified in this paper.

\section{Author Contributions}

Conception and design: Mastorakos, Ahn, Chen, Kellogg, Park. Acquisition of data: Mastorakos, Ahn. Analysis and interpretation of data: Mastorakos, Ahn. Drafting the article: Mastorakos, Ahn. Critically revising the article: all authors. Reviewed submitted version of manuscript: all authors. Approved the final version of the manuscript on behalf of all authors: Mastorakos. Statistical analysis: Mastorakos, Ahn. Administrative/technical/material support: Mastorakos, Ahn. Study supervision: Mastorakos, Sokolowski.

\section{Correspondence}

Panagiotis Mastorakos: University of Virginia Health System, Charlottesville, VA.pm5mt@hscmail.mcc.virginia.edu. 